\title{
An ab initio CASSCF study of Zero Field Splitting Fluctuations in the Octet Ground State of Aqueous [Gd(III)(HPDO3A)( $\left.\mathrm{H}_{2} \mathrm{O}\right)$ ]
}

Shehryar Khan, ${ }^{1}$ Rodolphe Pollet, ${ }^{2}$ Rodolphe Vuilleumier, ${ }^{3,4}$ Jozef Kowalewski, ${ }^{5}$ and Michael Odelius ${ }^{1, a}$

1) Department of Physics, Stockholm University, AlbaNova University Center, 10691 Stockholm, Sweden

2) NIMBE, CEA, CNRS, Université Paris-Saclay, CEA Saclay, 91191 Gif-sur-Yvette Cedex, France

3) PASTEUR, Département de chimie, École normale supérieure, UPMC Univ. Paris 06, CNRS, PSL Research University, 75005 Paris,

France

4) Sorbonne Universités, UPMC Univ. Paris 06, École normale supérieure, CNRS, Processus d'activation sélective par transfert d'énergie uni-électronique ou radiatif (PASTEUR), 75005 Paris, France

5) Department of Materials and Environmental Chemistry, Arrhenius Laboratory, Stockholm University, 10691 Stockholm, Sweden

(Dated: 2 December 2017) 
In this work we present ab initio calculations of the zero-field splitting (ZFS) of a gadolinium complex $\left[\mathrm{Gd}(\mathrm{III})(\mathrm{HPDO} 3 \mathrm{~A})\left(\mathrm{H}_{2} \mathrm{O}\right)\right]$ sampled from an ab initio molecular dynamics (AIMD) simulation. We perform both post-Hartree-Fock (complete active space self-consistent field - CASSCF) and density functional theory (DFT) calculations of the ZFS and compare and contrast the methods with experimental data. Two different density functional approximations (TPSS and LC-BLYP) were investigated. The magnitude of the ZFS from the CASSCF calculations is in good agreement with experiment, whereas the DFT results in varying degree overestimate the magnitude of the ZFS for both functionals and exhibit a strong functional dependence. It was found in the sampling over the ab initio molecular dynamics (AIMD) trajectory that the fluctuations in the transient ZFS tensor derived from DFT is not correlated with that of CASSCF, nor does the magnitude of the ZFS from CASSCF and DFT correlate. From the fluctuations in the ZFS tensor, we extract a correlation time of the transient ZFS which is on the sub-picosecond time scale, showing a faster decay than experimental estimates.

a)Electronic mail: odelius@fysik.su.se; http://www.fysik.su.se/〜 odelius/ 


\section{INTRODUCTION}

Gd(III) complexes are efficient contrast agents for magnetic resonance imaging(MRI) due to the fact that they enhance the longitudinal and transverse relaxation rates of neighboring protons in solution ${ }^{1 / 3}$. The drastic effect of electron spin relaxation on the reduction of paramagnetic relaxation enhancement(PRE) of nuclear relaxation was first explained by Bloembergen and Morgan ${ }^{4}$. The relaxation can be described by solving for the time-evolution of the electron spin, with a spin Hamiltonian containing a Zeeman term, describing the interaction with the external magnetic field, and a ZFS term, related to the electronic structure and molecular structure around the paramagnetic center. The electron spin relaxation in Gd(III) is essentially driven by fluctuations in the ZFS, which is caused by second-order effects of the spin-orbit coupling. Until the 1980 's the electron spin relaxation times $\left(\mathrm{T}_{1 e}\right.$ and $\mathrm{T}_{2 e}$ ) were computed on the basis of the analytic Redfield equations, which relies on the transient fluctuations in the zero-field splitting (ZFS) ${ }^{5}$, but it was discovered for the case of Gd(III) that the average ZFS in the molecular frame (the static ZFS) plays a significant role in influencing these relaxation times ${ }^{6 / 7}$. Therefore, to understand electron spin relaxation completely, both the static ZFS and instantaneous deviations from the static ZFS (the transient ZFS) need to be investigated.

The ZFS spin Hamiltonian of the octet ground state of the Gd(III) complex is described by an approximate $2^{\text {nd }}$ order expression:

$$
H_{Z F S}=\hat{S} \cdot \overline{\bar{D}} \cdot \hat{S}
$$

in which $\overline{\bar{D}}$ is the zero field splitting tensor and $\hat{S}$ is the electron spin angular momentum operator. For the octet state in $\operatorname{Gd}(\mathrm{III})$, higher $\left(4^{\text {th }}\right.$ and $\left.6^{\text {th }}\right)$ order terms can contribute, but are neglected in the ZFS spin Hamiltonian in Eq. 1.

In the treatment of the ZFS, we need to consider three reference frames. The laboratory frame $(\mathrm{L})$, in which the external magnetic field is applied. The ZFS in the laboratory is modulated by reorientation of the $\mathrm{Gd}(\mathrm{III})$ cluster, whose structure defines a molecular frame (M). The ZFS calculations presented below will be performed in the molecular frame. Finally, since $\overline{\bar{D}}$ is a symmetric traceless tensor it is convenient to determine the principal axis frame $(\mathrm{P})$, in which the tensor is diagonal and can be described by its cylindrical $D$ and rhombic $E$ components. 


$$
\hat{H}_{Z F S}^{(P)}=D\left[\hat{S}_{z}^{2}-\frac{1}{3} S(S+1)\right]+E\left[\hat{S}_{x}^{2}-\hat{S}_{y}^{2}\right]
$$

The $D$ and $E$ parameters are extracted from the diagonal elements of the $\overline{\bar{D}}$ tensor in the principal axis frame:

$$
\begin{aligned}
D & =D_{z z}^{(P)}-\frac{1}{2}\left(D_{x x}^{(P)}+D_{y y}^{(P)}\right)=\frac{3}{2} D_{z z}^{(P)}, \\
E & =\frac{1}{2}\left(D_{x x}^{(P)}-D_{y y}^{(P)}\right)
\end{aligned}
$$

where the Cartesian components are defined to fulfill $0 \leq E / D \leq 1 / 3$, thus making $D$ the dominant contribution. The ZFS in the molecular frame is modulated both by variations in the magnitude of the $D$ and $E$ components and by reorientation of the principle axis frame relative to the molecular frame.

We will employ the conventional method of dealing with the total ZFS in the molecular frame $(\mathrm{M})$, as described above, by dividing it into two parts: the static ZFS, which is the time-average of the ZFS Hamiltonian, and the transient ZFS, describing the instantaneous deviation from the static spin Hamiltonian. Therefore, the total ZFS Hamiltonian can be described as a summation of the two terms.

$$
\hat{H}_{Z F S}^{(M)}(t)=\hat{H}_{Z F S, \text { static }}^{(M)}+\hat{H}_{Z F S, \text { trans }}^{(M)}(t)
$$

The time-dependent transient tensor is obtained by subtracting the static $\overline{\bar{D}}$ static tensor from each element of the instantaneous $\overline{\bar{D}}$ tensor:

$$
\overline{\bar{D}}_{\text {trans }}=\left(\begin{array}{lll}
d_{11} & d_{12} & d_{13} \\
d_{21} & d_{22} & d_{23} \\
d_{31} & d_{32} & d_{33}
\end{array}\right)
$$

Parameters extracted from each of the two terms in Eq. 4 can subsequently be used as a basis for describing both electronic spin relaxation and nuclear spin relaxation. A common, simple expression for the longitudinal electron spin relaxation rate has been proposed by Belorizky and Fries ${ }^{8}$ :

$$
\frac{1}{T_{1 e}}=\frac{12}{5} \Delta_{S}^{2} \tau_{2}\left(\frac{1}{1+\omega_{0}^{2} \tau_{2}^{2}}+\frac{4}{1+4 \omega_{0}^{2} \tau_{2}^{2}}\right)+\frac{12}{5} \Delta_{T}^{2} \tau_{v}\left(\frac{1}{1+\omega_{0}^{2} \tau_{v}^{2}}+\frac{4}{1+4 \omega_{0}^{2} \tau_{v}^{2}}\right)
$$


Here, the symbol $\Delta_{S}$ stands for the magnitude of the static ZFS, defined as:

$$
\Delta_{S}=\sqrt{2 / 3 D_{S}^{2}+2 E_{S}^{2}}
$$

which can be extracted in the molecular frame from the $\overline{\bar{D}}$ tensor.

The static contribution $\Delta_{S}$ is calculated from the principal frame $D_{S}$ and $E_{S}$ of the mean tensor (averaged in the molecular frame). The corresponding transient contribution $\Delta_{T}$ is originally introduced in the so-called pseudo-rotation model ${ }^{9 \mid 10}$, where the distortions of the complex are assumed to yield a ZFS of constant magnitude and variable orientation of its principal axes. The symbol $\tau_{2}$ denotes the rotational correlation time for the rank- 2 spherical harmonics of the complex and $\tau_{v}$ refers to a corresponding correlation time for the reorientation of the principal direction of the transient ZFS. In turn, $\tau_{2}$ can be related to the rotation diffusion constant through $\tau_{2}=1 /\left(6 D_{R}\right)$. Finally $\omega_{0}$ is the electron spin Larmor frequency. The relation between the $\Delta_{T}$ and the elements of the matrix in Eq. 5 has been discussed in our earlier work 11112 .

Notice that we will consistently use the $D$ and $E$ parameters for the total ZFS in the principal axis frame of the $\overline{\bar{D}}$ tensor. whereas $D_{S}$ and $E_{S}$ refer to the $\overline{\bar{D}}_{\text {static }}$ tensor and $D_{T}$ and $E_{T}$ to the $\overline{\bar{D}}_{\text {trans }}$ tensor. All of which, might have independent principal axis frames.

Dynamical simulations when used in combination with quantum chemical computations become a powerful tool to probe both static and time-dependent properties of molecules. A combined approach makes it possible to extract both $\overline{\bar{D}}_{\text {static }}^{(M)}$ and $\overline{\bar{D}}_{\text {trans }}^{(M)}(t)$, the reorientation of the molecular frame and correlations with molecular degrees of freedom. If accurate simulations are performed with sufficient sampling, we can acquire all information needed to model both the electron spin relaxation and the nuclear spin relaxation from first principles.

In the nineties, a few attempts of first principle derivation of spin relaxation were performed for aqueous $\mathrm{Ni}(\mathrm{II})^{11112}$ combining classical molecular dynamics (MD) and a simple ab initio method for calculation of the ZFS, where time correlation fluctuations in the ZFS were reported. Later a model was developed for electron spin relaxation ${ }^{13}$ through studying the fluctuations of the transient ZFS, obtained from solving the Smoluchowski equation for diffusion. More recently, AIMD simulations and density functional theory (DFT) calculations have been performed on aqueous $\mathrm{Ni}(\mathrm{II})^{14}-17$ to report on the dynamical characteristics of the ion in solution and its paramagnetic shielding parameters. Furthermore, the same group has also performed complete active space self-consistent field (CASSCF) based studies 
to report on the paramagnetic shielding parameters of $\mathrm{Ni}(\mathrm{II})$ and other magnetic centers 18 .

An investigation of gadolinium 1,4,7-tris(carboxymethyl)-10-(2'-hydroxypropyl)-1,4,7,10tetraazacyclo-dodecane $\left[\mathrm{Gd}(\mathrm{III})(\mathrm{HPDO} 3 \mathrm{~A})\left(\mathrm{H}_{2} \mathrm{O}\right)\right]$ based on structures sampled from ab initio molecular dynamics(AIMD) and transient ZFS calculated with DFT was performed by Lasoroski et al! 19 . In their work they showed that the transient ZFS matrix elements $d_{i j}$ had nearly Gaussian distributions over the trajectory and were pairwise uncorrelated. The $\left[\mathrm{Gd}(\mathrm{III})(\mathrm{HPDO} 3 \mathrm{~A})\left(\mathrm{H}_{2} \mathrm{O}\right)\right]$ complex is presented in Fig. 1. They also showed that the static ZFS was primarily influenced by an extended ligand (in exchange for the attached water molecule as shown in Fig. If and that the correlation time and frequency from the transient contribution were not greatly effected by the elongation of the ligand. However, the DFT calculations resulted in a magnitude $\left(\Delta_{S}\right)$ of the static ZFS that deviated from experimental estimates 20 .

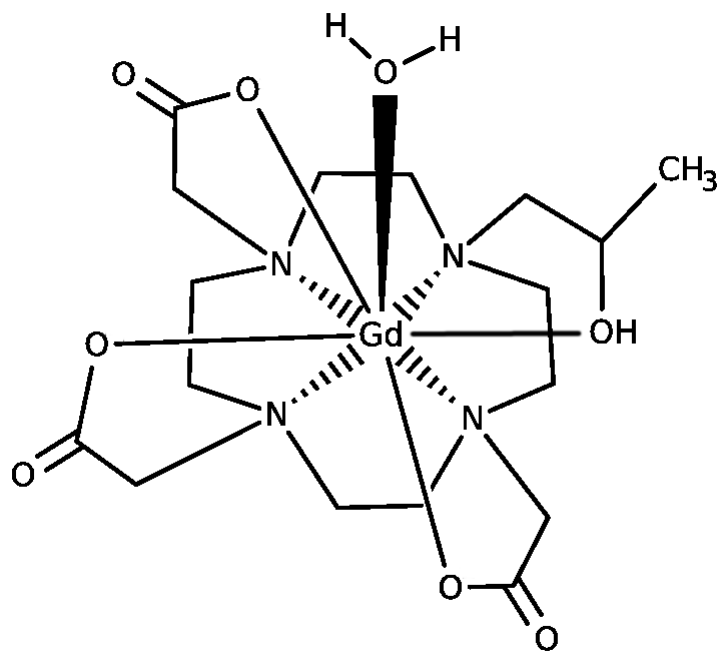

FIG. 1. A schematic picture of gadolinium 1,4,7-tris(carboxymethyl)-10-(2'-hydroxypropyl)1,4,7,10-tetraazacyclo-dodecane[Gd(III) $\left.(\mathrm{HPDO} 3 \mathrm{~A})\left(\mathrm{H}_{2} \mathrm{O}\right)\right]$ contrast agent showing the 9-fold coordination around the $\mathrm{Gd}(\mathrm{III})$ ion.

The current paper is an extension of the work by Lasoroski et al! ${ }^{19}$ by using configurations from the same trajectory and performing the ZFS calculations with post-Hartree-Fock methods (CASSCF), which have been shown to provide static ZFS parameters that are more reliable than those from $\mathrm{DFT}^{21}$. The current paper also investigates the relationship between the ZFS obtained from CASSCF calculations and from DFT calculations, where the 
latter were reproduced using the same methodology as in the previous study $\underline{19}$. The paper is organized as follows: First a test for basis sets was performed, by comparing QZVP and TZVP for a limited sampling of the complex and thereby establish the stability of the results from the CASSCF calculations. Next, the fluctuations in the splitting of the octet ground state in the Kramer doublet energy levels and the evolution of the $D$ parameter over time are presented and contrasted to DFT results followed by the distributions for the static and transient ZFS parameters. The time correlation functions of the transient ZFS are presented for both CASSCF, DFT and compared to the previous computations. Finally, a discussion on the accuracy of DFT versus CASSCF is given. We evaluate whether long-range corrected functionals in DFT provide more reasonable static properties, an investigation motivated by them being computationally much more economical than post-Hartree-Fock methods. The results refer to the CASSCF method unless mentioned otherwise.

\section{COMPUTATIONAL AND THEORETICAL DETAILS}

All ZFS calculations were carried out using the ORCA software ${ }^{22}$ version 3.3. The AIMD trajectory configurations utilized for this study were the same as used in the previous study by Lasoroski et $a^{119}$, consisting of a single $\mathrm{Gd}(\mathrm{HPDO} 3 \mathrm{~A})\left(\mathrm{H}_{2} \mathrm{O}\right)$ complex in a $15.4 \AA$ cubic box with 98 solvent water molecules. The configuration space sampling consisted of 4 blocks $(90 \mathrm{x} 4$ snapshots) of $0.57 \mathrm{ps}$ each. The details of the simulation have been extensively described in the previous investigation 19 .

Calculations of lanthanide complexes require an accurate description of relativistic effects. To do this there are two methods that can be employed, an all-electron relativistic treatment or an implicit treatment of the inner electron shell, by inclusion of relativistic effective core potentials or pseudo-potentials. Here we use an explicit all-electron relativistic method that utilizes the Douglas-Kroll-Hess(DKH) ${ }^{23}$ formulation and atomic mean field integrals ${ }^{24}$ for the CASSCF runs with the subsequent inclusion of spin-orbit coupling. The CAS space consisted of 7 electrons active over 7 orbitals, which acquire $4 f$ character. The CASSCF calculations were state-averaged over the ground octet state and 48 excited sextet states, and then the spin-orbit coupled matrix over the pure multiplicities was diagonalized to produce the final complex states and the ZFS of the octet ground state.

For choice of basis set we ran tests on a limited sampling from snapshots of the AIMD 
using the QZVP 25 and def2-TZVPP ${ }^{26}$ basis sets on the ligands. The SARC-DKH ${ }^{27}$ basis was incorporated on the central Gd(III) ion. The comparison of the splitting of the energy levels of the highest and lowest ground state energy produced from each of these basis sets is shown in Appendix VI. After evaluating the def2-TZVPP results against the QZVP results, the def2-TZVPP basis sets were eventually used for all the calculations on the ligand atoms. Another test that was performed was the accuracy of using auxiliary basis sets $(\mathrm{QZVP} / \mathrm{j}$ and def2-TZVPP $/ \mathrm{j}$ ) with the RIJCOSX ${ }^{28}$ approximation and the results varied quite significantly so that use of auxiliary basis sets was deemed unreliable for calculations of ZFS parameters within the CASSCF method.

For the DFT calculations the all-electron Zeroth Order Regular Approximation(ZORA) 29 was used with the TPSS functional and the SVP basis set as described in the earlier study by Lasoroski et a 19 . Furthermore for the direct comparison of ZFS from DFT with the CASSCF method ${ }^{30}$ both Couple-Perturbed $(\mathrm{CP})^{31}$ and Pederson-Khanna(PK) ${ }^{32}$ formalisms were employed and the results described in this paper refer to the CP method. For further investigations the long-range corrected LC-BLYP functional was used as well.

\section{RESULTS AND DISCUSSION}

\section{A. Time evolution of the ZFS}

In Fig. 2, we present the correlation between the octet Kramer doublet levels and the $D$ parameter for the CASSCF sampled over the AIMD trajectory. There is a nearly linear relationship between the $D$ parameter and the energy levels arising from the splitting of the ground state. The limited influence of the rhombic $E$ parameter is seen in the small spread of the higher Kramer doublet levels. The clear correlation between the $D$ parameter and the splitting in the Kramer doublets is also taken as an indication of the validity of the approximate ZFS spin Hamiltonian in Eq. 1. The relative splitting between the Kramer doublets of the ground state octet determines the sign of the $D$ parameter. In this case it forms a negative $D$ parameter. The values of $D$ vary from $-0.10 \mathrm{~cm}^{-1}$ to $-0.02 \mathrm{~cm}^{-1}$, and the splitting of the highest ground state octet varies from $0.40 \mathrm{~cm}^{-1}$ to $1.20 \mathrm{~cm}^{-1}$. Fig. 3 a shows the splitting of the ground state octet energy levels and Fig. 3b shows the $D$ parameter evolve over a single block of 0.57 ps. For the $D$ parameter the results from the DFT calculation 
are also shown. There is a very weak correlation between the variations in the $D$ parameter from CASSCF and DFT as can be seen in Fig. $3 \mathrm{~b}$, in the occurrence of particularly large amplitude crests and troughs, but otherwise the correlation is negligible. Fig. 4 shows the distribution of the $D$ parameter and the $E$ parameter from the CASSCF calculations over the entire trajectory consisting of four blocks, each of $0.57 \mathrm{ps}$. We observe a significant spread in magnitude of the cylindrical $D$ parameter, but the rhombic $E$ component is generally small. In Fig. 5, a sampling of the orientation of the principal axis system is presented. It shows that the cylindrical component $D$ of the total ZFS tensor (corresponding to the eigenvalue of largest magnitude $\left.D_{z z}^{(P)}\right)$ is related to an eigenvector which exhibit considerable orientational fluctations around a direction approximately in the plane of nitrogen atoms. The eigenvectors corresponding to $D_{x x}^{(P)}$ and $D_{y y}^{(P)}$ reorient even more since the tensor is closely cylindrical.

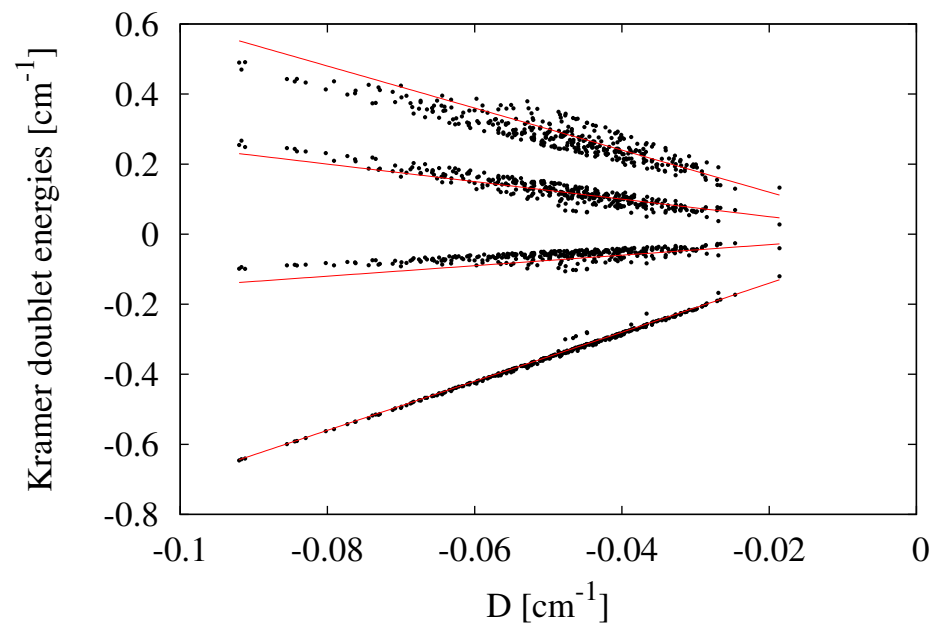

FIG. 2. Relationship between the $D$ parameter(x-axis) and the relative energies of Kramer doublet ground state energy levels(y-axis)

\section{B. Comparison between DFT and CASSCF of the transient ZFS}

In Fig. 6, we plotted the correlation of the CASSCF and DFT results of each $d_{i j}$ element in the $\overline{\bar{D}}_{\text {trans }}$ tensor. The magnitude of the CASSCF and DFT results clearly differs, but more importantly only some of the $d_{i j}$ element show any correlation between the two methods. Hence, assuming that the CASSCF results are more trustworthy, the DFT method using 

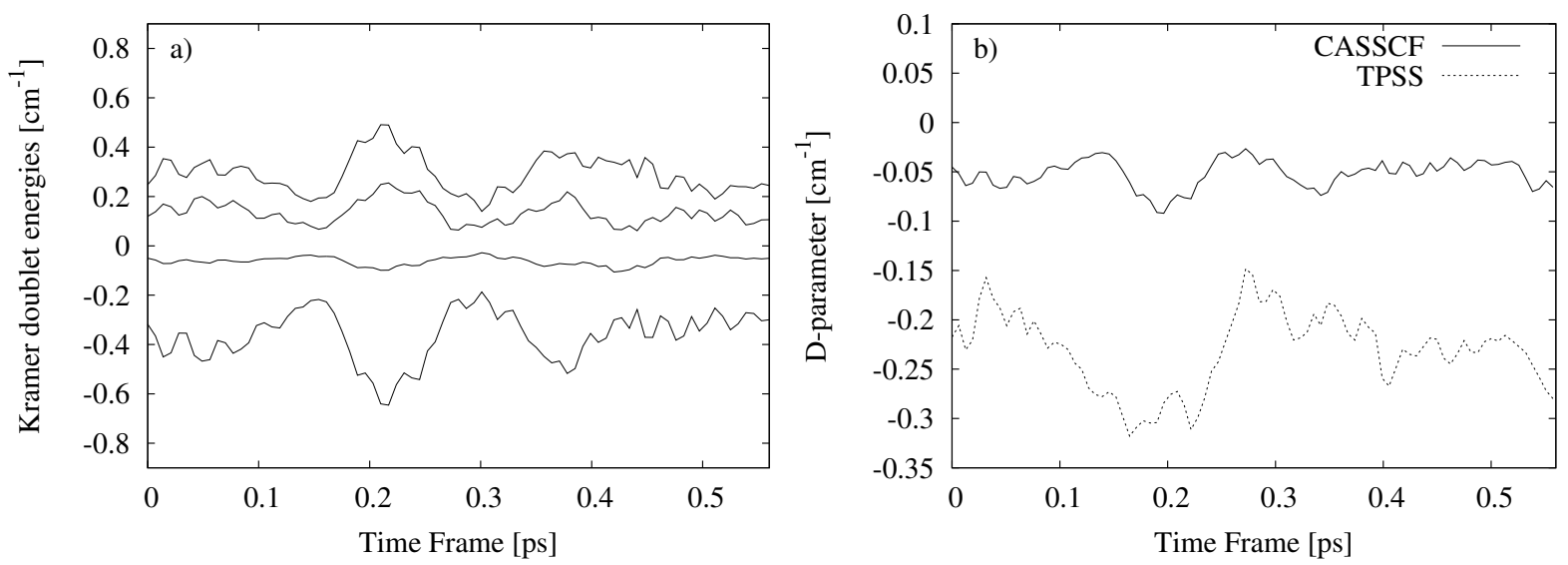

FIG. 3. Time evolution of the splitting of the octet ground state into Kramer doublets using CASSCF (left) and evolution of the $D$ parameter using CASSCF and DFT(right) sampled along the AIMD trajectory for the hydrated $\left[\mathrm{Gd}(\mathrm{III})(\mathrm{HPDO} 3 \mathrm{~A})\left(\mathrm{H}_{2} \mathrm{O}\right)\right]$.
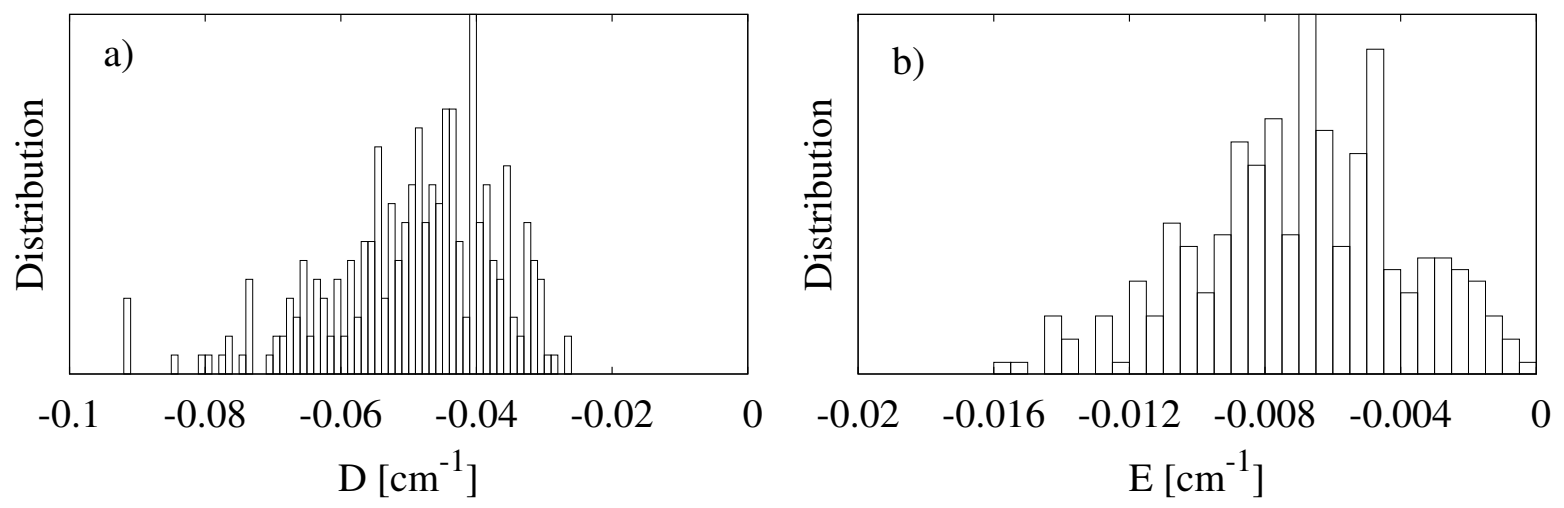

FIG. 4. Distribution of ZFS parameters $D$ and $E$ sampled over the AIMD simulation.

the TPSS functional is not reliable for studying the molecular origin of fluctuations in the ZFS Hamiltonian and for establishing the basis for spin dynamics simulations. Comparing the $d_{i j}$ elements of CASSCF with the PK method gives a Pearson coefficient of 0.078 and a 2-tailed p-value of 0.15 . The Pearson value allows us to see how two sets of data are correlated, the value 1 being perfect linear correlation , -1 being anti-correlation and 0 being no relationship at all. The same is true for the CP method, as is shown in Fig. 6 there is only a weak correlation for some of the components. Therefore, as far as the $\overline{\bar{D}}_{\text {trans }}$ tensor is concerned there is no correlation between the two methods under the given conditions.

From the comparison in Fig. 7 of the $D$ parameter between CASSCF and the TPSS 


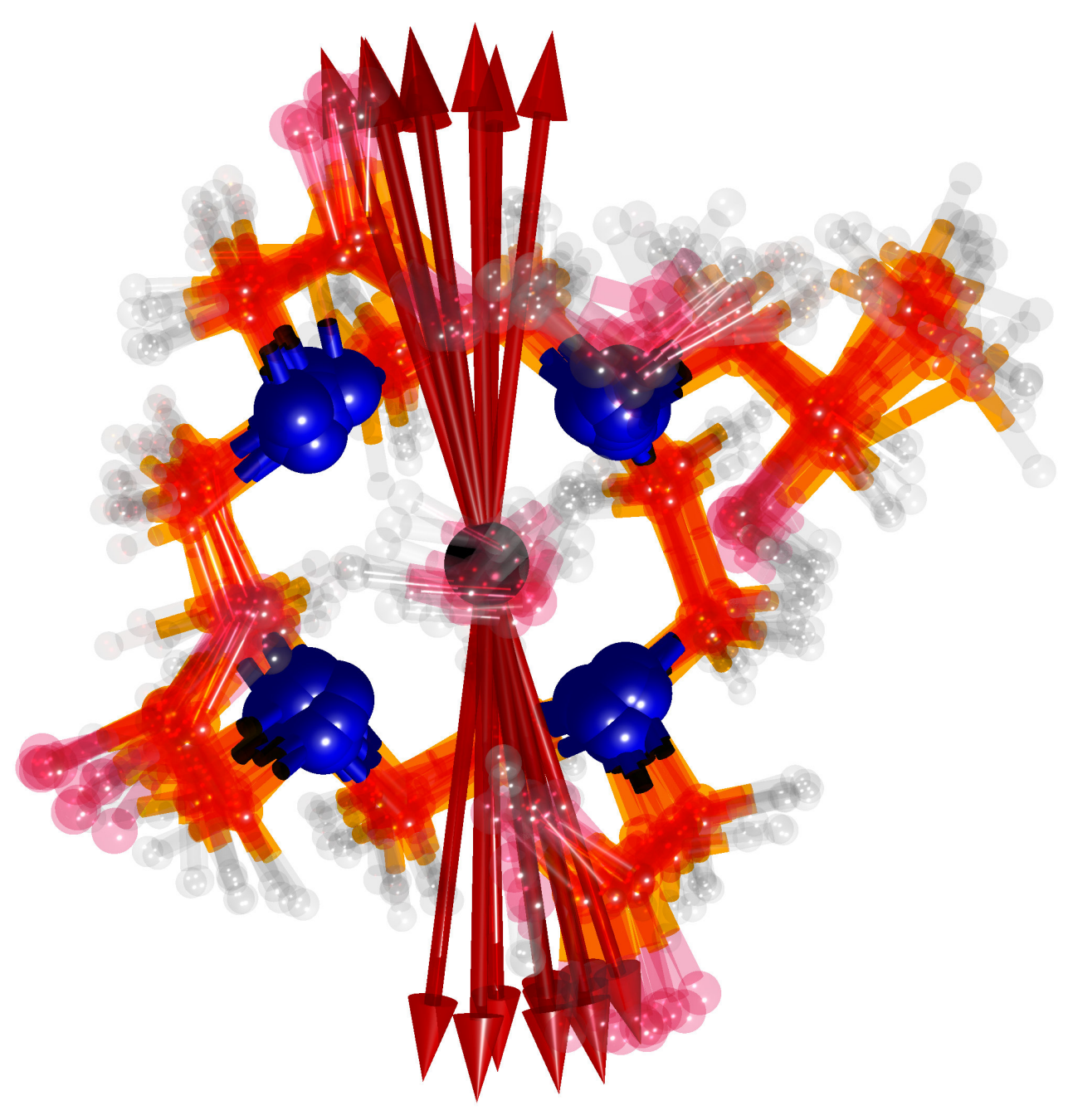

FIG. 5. The eigenvectors corresponding to the largest component $\left(D_{z z}^{(P)}\right)$ of the principal axis system of the total ZFS tensor are superimposed for 10 AIMD configurations. The average orientation of the complex is that of Fig. 1, viewed from a direction normal to the plane of nitrogen atoms.

functional using DFT we see that the values range from $-0.12 \mathrm{~cm}^{-1}$ to $-0.28 \mathrm{~cm}^{-1}$ in the case of the TPSS functional and from $-0.02 \mathrm{~cm}^{-1}$ to $-0.10 \mathrm{~cm}^{-1}$ in the case of CASSCF. The static values using CASSCF and DFT are shown in Table I. The $D_{S}$ and $E_{S}$ of the static ZFS tensor does not necessarily correspond to the means of the distributions of $D$ and $E$, 
TABLE I. Static ZFS parameters in $\mathrm{cm}^{-1}$ of $\mathrm{Gd}(\mathrm{HPDO} 3 \mathrm{~A})\left(\mathrm{H}_{2} \mathrm{O}\right)^{-}$

\begin{tabular}{cccc}
\hline \hline & $\mathrm{D}_{S}$ & $\mathrm{E}_{S} / \mathrm{D}_{S} \Delta_{S}\left[\mathrm{rad} \mathrm{s}^{-1}\right]$ \\
\hline CP-TPSS $^{*}$ & -0.296 & 0.087 & $4.60 \times 10^{10}$ \\
PK-TPSS $^{*}$ & -0.200 & 0.093 & $3.12 \times 10^{10}$ \\
LC-BLYP & -0.069 & 0.182 & $1.12 \times 10^{10}$ \\
CASSCF & -0.048 & 0.16 & $0.77 \times 10^{10}$ \\
Exp. & & & $0.99 \times 10^{10}$ \\
\hline
\end{tabular}

${ }^{*}$ Values obtained by Lasoroski et al! ${ }^{19}$

respectively. The static value from the DFT calculation using the TPSS functional gives $-0.296 \mathrm{~cm}^{-1}$ and CASSCF gives a static value of $-0.048 \mathrm{~cm}^{-1}$ and an average $\mathrm{E} / \mathrm{D}$ value of 0.16. For CASSCF, the magnitude of the static ZFS $\left(\Delta_{S}\right)$ gives a value of $0.77 \times 10^{10} \mathrm{rad}$ $\mathrm{s}^{-1}$ compared to the experimental value of $0.99 \times 10^{10} \mathrm{rad} \mathrm{s}^{-1}$. In comparison the TPSS method predicted a value of $4.6 \times 10^{10} \mathrm{rad} \mathrm{s}^{-1}$.

It has been suggested that long-range functionals provide better magnetic properties ${ }^{33}$. These functionals separate the electron-electron interaction into two parts, the long-range and the short-range ${ }^{34 / 35}$ We used the LC-BLYP functional on a single block of $0.57 \mathrm{ps}$ and computed the ZFS values for 90 snapshots. The $D_{S}$ parameter of the static ZFS was significantly improved from $-0.296 \mathrm{~cm}^{-1}$ (for TPSS) to $-0.069 \mathrm{~cm}^{-1}$ (for LC-BLYP) compared to the CASSCF value of $-0.048 \mathrm{~cm}^{-1}$. However, there is still little correlation between CASSCF and any of the DFT approximations as can be seen from Fig. 7b , which indicates that none of the DFT approximations captures the correct physics.

\section{The Distributions of the transient ZFS}

Apart from the reorientation of Gd(III) complex and the associated modulation in the static spin Hamiltonian $\hat{H}_{Z F S, \text { static }}^{(L)}$, the variations in magnitude and orientation due to fluctuations in the time-dependent transient contribution $\hat{H}_{Z F S \text { trans }}^{(M)}(t)$ control the spin dynamics. Fig. 8 shows the distribution of the individual $d_{i j}$ components of the $\overline{\bar{D}}_{\text {trans }}$ matrix for the CASSCF calculation sampling configurations over the four blocks. The CASSCF results are an order of magnitude smaller than the DFT results presented in the study of Lasoroski et 

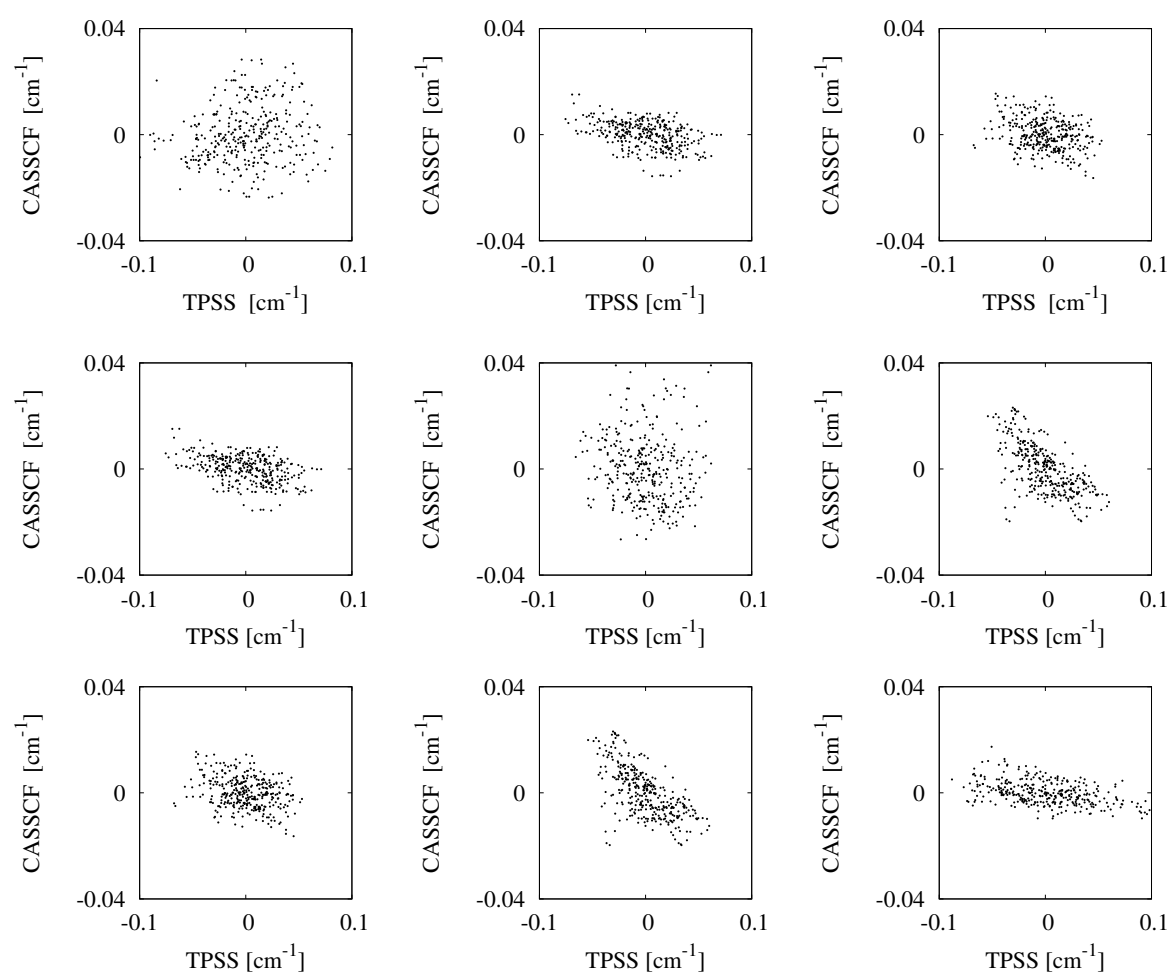

FIG. 6. Correlation of transient $d_{i j}$ matrix elements from CASSCF and DFT calculation sampled over the AIMD simulation.
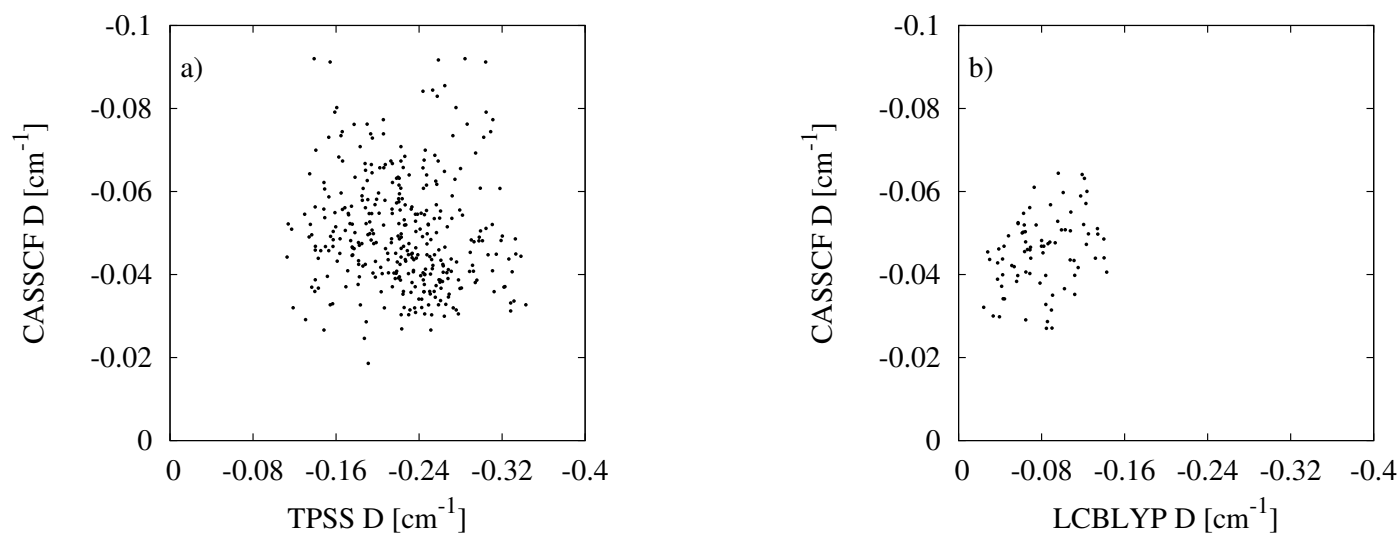

FIG. 7. a) Correlation of total $D$ parameter between CASSCF and DFT calculations sampled over the AIMD simulation. b) A comparison of the total $D$ parameter using the LC-BLYP functional with configurations taken from a single block of 0.57 ps compared to the corresponding CASSCF geometries.

$a_{\underline{119}}^{19}$, but also display roughly Gaussian distributions. The elements are symmetric $d_{i j}=d_{j i}$ 
by necessity, hence only $d_{i j}$ for $j \geq i$ are displayed. However, the CASSCF results, presented in Fig. 8 in our study are less uniform than the DFT results presented by Lasoroski et a $a^{119}$. For example, the elements $d_{11}$ and $d_{22}$ show broader distributions than the other elements in the transient $\overline{\bar{D}}_{\text {trans }}^{(M)}$ tensor. This is influenced by the limited sampling of course, but is also related to the fact that we are studying the tensor in the molecular frame of the $\left[\mathrm{Gd}(\mathrm{III})(\mathrm{HPDO} 3 \mathrm{~A})\left(\mathrm{H}_{2} \mathrm{O}\right)\right]$ complex, in which there might be orientational dependences.
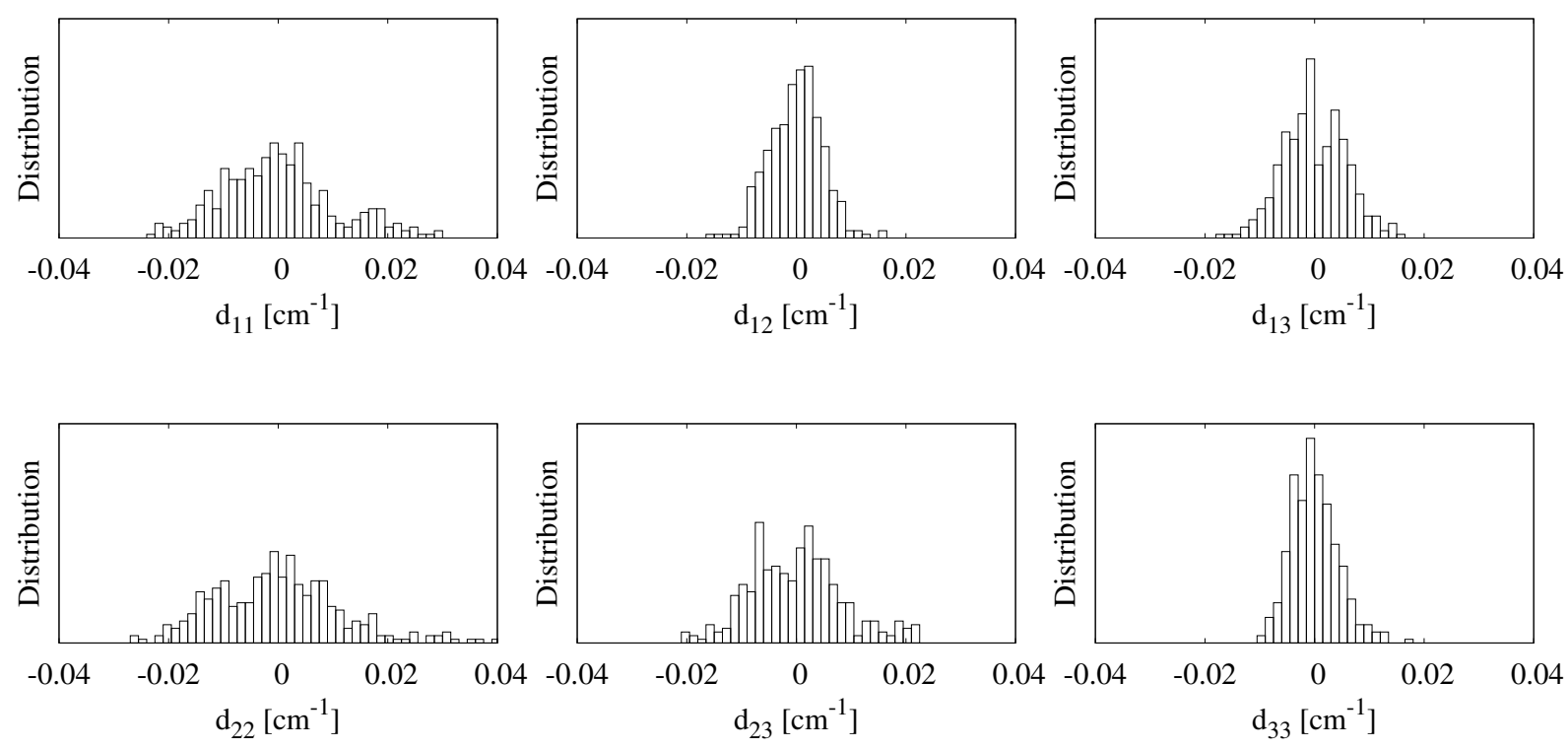

FIG. 8. Distribution of the $d_{i j}$ components of the $D_{Z F S \text {,trans }}$ for the CASSCF calculation sampling configurations over the AIMD simulation(Top: $d_{11}, d_{12}, d_{13}$. Bottom: $d_{22}, d_{23}, d_{33}$ )

\section{Correlations in the transient ZFS matrix elements $d_{i j}$}

Fig. 9 shows the correlation between individual elements of the $\overline{\bar{D}}_{\text {trans }}$ matrix. There is clearly more of a correlation between some of the individual $d_{i j}$ elements for the CASSCF results than for the TPSS functional in DFT, where the largest Pearson value obtained was -0.4 for the elements $\left(d_{11}, d_{22}\right)$.

For CASSCF the diagonal elements $\left(d_{11}, d_{22}\right)$ and $\left(d_{22}, d_{33}\right)$ exhibit a linear correlation. This seems to be due to the fact that the transient matrix element $d_{33}$ remains close to a value of 0 . Since the ZFS tensor is traceless, there is a correlation in $\left(d_{11}, d_{22}\right)$ which compensates for the nearly vanishing $d_{33}$. The Pearson values for $\left(d_{11}, d_{22}\right),\left(d_{11}, d_{33}\right)$ and 
$\left(d_{22}, d_{33}\right)$ are $0.55,-0.51,-0.70$, respectively. The remaining pair-wise correlations of $d_{i j}$ elements show a Pearson value below 0.4. The much smaller magnitude of the fluctuations in the CASSCF results as compared to the DFT results, using the TPSS functional, is also apparent.
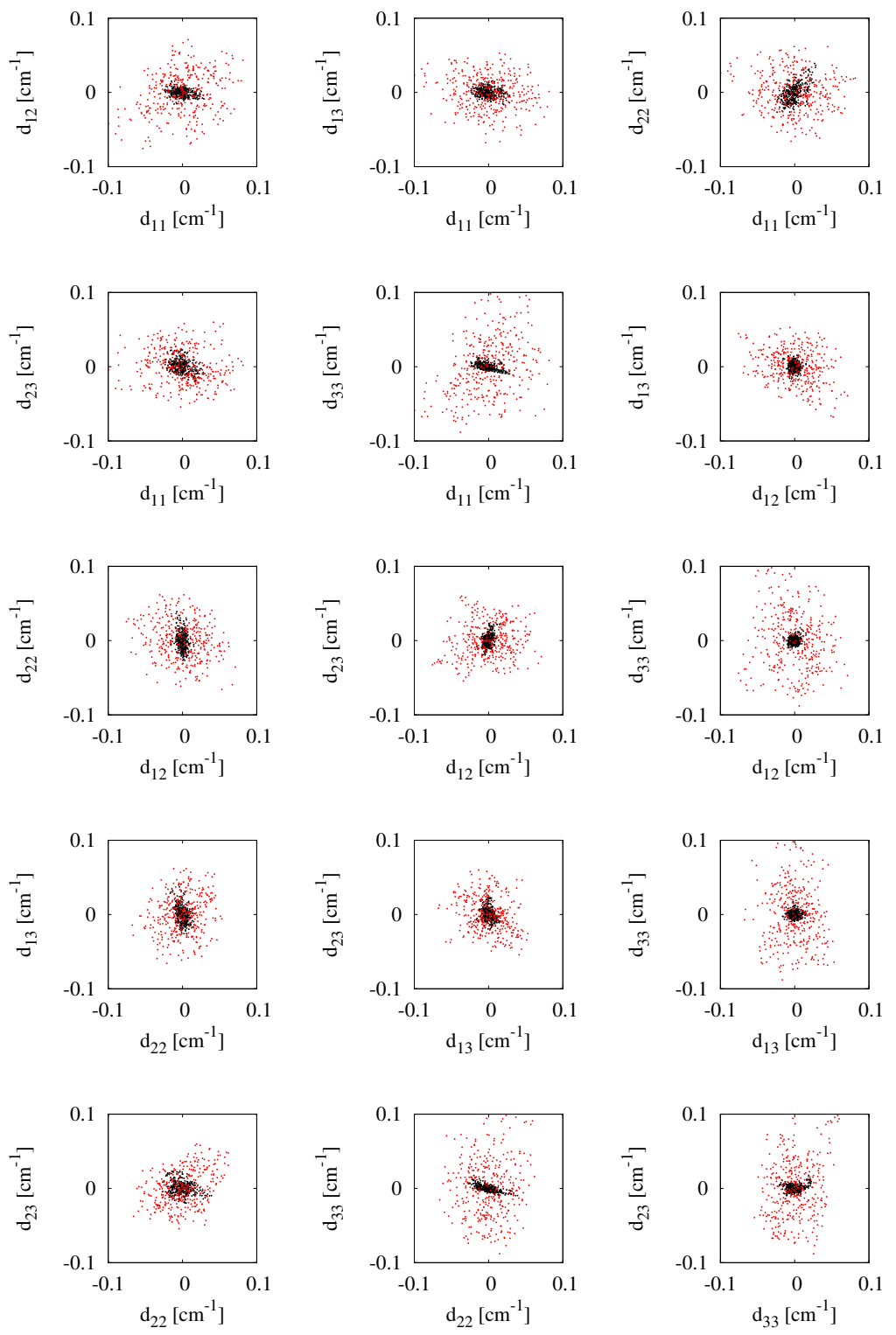

FIG. 9. Pair-wise correlations between different transient $d_{i j}$ components of the matrix $\overline{\bar{D}}_{\text {trans }}$ for the CASSCF calculations(black dots), and for DFT calculations(red dots) sampling over the AIMD simulation and using the same DFT parameters as in Lasoroski et al 19 . 


\section{E. Time correlation functions of the fluctuations in the $\overline{\bar{D}}_{\text {trans }}(\mathbf{t})$ tensor}

From the time correlation function $(\mathrm{TCF})$ of the $\overline{\bar{D}}_{\text {trans }}(\mathrm{t})$ tensor, we can calculate a characteristic time-scale of the fluctuations modulating the spin dynamics. A way to improve the statistics is to assume that $d_{i j}$ elements are uncorrelated, despite the results of the counterpart presented above, and average over the TCFs of the individual matrix elements, $\mathrm{C}(\mathrm{t})$. The average of the normalized auto-correlation function has been computed using the nine coefficients of the transient ZFS:

$$
C(t)=\frac{1}{9} \sum_{i, j} \frac{\left\langle d_{i j}(0) d_{i j}(t)\right\rangle}{\left\langle d_{i j}(0) d_{i j}(0)\right\rangle}
$$

We also included the full TCF by using the equation:

$$
C(t)_{f u l l}=\frac{\left\langle\sum_{i, j} d_{i j}(0) d_{i j}(t)\right\rangle}{\left\langle\sum_{i, j} d_{i j}(0) d_{i j}(0)\right\rangle}
$$

Fig. 10 shows $C(t)$ derived from the CASSCF and DFT results differ, apart from the initial decay which occur on a similar time-scale. There is a slight discrepancy between our CPTPSS results and those obtained from Lasoroski et al. due to the fact that we used 4 blocks rather than 5 blocks of data for our analysis. However beyond 0.1 ps the CASSCF and DFT curves differ. We can fit our CASSCF data to an oscillatory and exponentially decaying curve of the form $\exp \left(-\mathrm{t} / \tau_{c}\right) \cos (2 \pi \nu \mathrm{t})$ which gives a correlation time, $\tau_{c}$ of 63.8 fs and characteristic frequency $\nu$ of $199.5 \mathrm{~cm}^{-1}$.

This correlation time is shorter than that anticipated from the CP method using the TPSS functional which gives a correlation time and frequency of $95.3 \mathrm{fs}$ and $171.82 \mathrm{~cm}^{-1}$ as computed by Lasoroski et al.19, but falls on the same scale of values. Furthermore, the error bars for the CASSCF are rather large as can be seen in the figure so the correlation time obtained from the DFT computations could easily be justified. But we need to keep in mind the CASSCF and DFT are only weakly correlated, so the physical meaning of the DFT results might be questioned. To conclude the investigations of the fluctuations in the ZFS, we also calculated TCFs of the instantaneous deviation in the $D$ parameter. Hence, we are investigating the fluctuations in the $D$ parameters of the total ZFS, as a means to separately study only the fluctuations in the magnitude of the ZFS, without contributions from the reorientation of the principal axis system $(\mathrm{P})$. Notice, however that this is not identical to

fluctuations in magnitude of the transient ZFS, since the principal axis frames of the $\overline{\bar{D}}$ and 
$\overline{\bar{D}}_{\text {trans }}$ tensors do not necessarily coincide. In Fig. 11, we present the normalized TCF of the deviation in the $D$ parameter. We found that the TCF for the rhombic terms and the cross-terms between the rhombic and axial contributions were zero within the error margins. A fitting of the TCF in Fig. 11 using the curve $\exp \left(-\mathrm{t} / \tau_{c}\right) \cos (2 \pi \nu \mathrm{t})$, gives a correlation time, $\tau_{c}$ of $61.7 \mathrm{fs}$ and characteristic frequency $\nu$ of $222.1 \mathrm{~cm}^{-1}$, which shows that the decay of $\mathrm{X}(\mathrm{t})$ is on the same scale as $C(t)$.
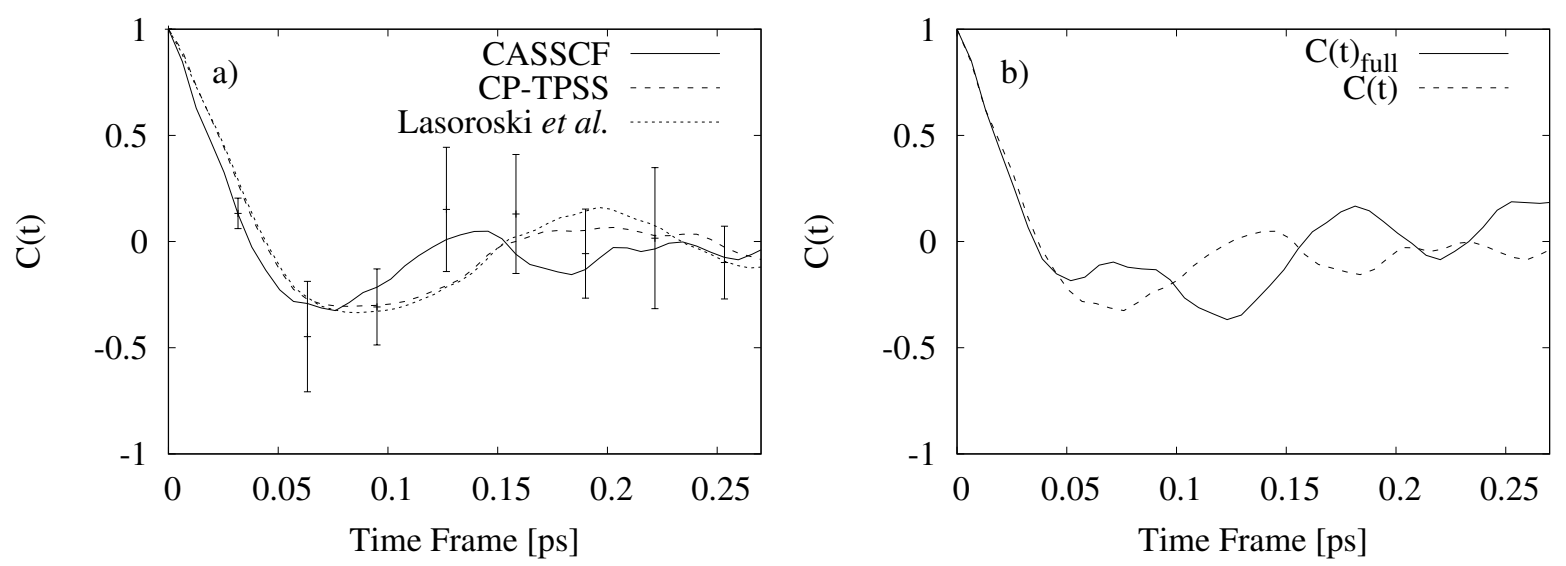

FIG. 10. (a) Time correlation function $\mathrm{C}(\mathrm{t})$ of the transient ZFS tensor, as defined in Eq. 8 . The labels CASSCF(black solid) and DFT(red dashed) refers to calculations in the present study and DFT[Lasoroski JCP 2014](blue dotted) is reproduced from Fig. 5 in Lasoroski et al.[19] (b) Comparison of $\mathrm{C}(\mathrm{t})$ and $\mathrm{C}(\mathrm{t})_{f u l l}$, as defined in Eqs. 8 and 9 .

\section{DISCUSSION}

The phenomenon of paramagnetic relaxation enhancement of nuclear spins around $\mathrm{Gd}(\mathrm{III})$ contrast agents is to a large extent dominated by relaxation of the electronic spin, which interacts strongly with the nuclear spins. The electronic spin relaxation is influenced by both the static and transient ZFS, through four main parameters: $\Delta_{S}$ (magnitude of the static ZFS), $\Delta_{T}$ (magnitude of the transient ZFS), $\tau_{2}$ (correlation time of the Brownian rotational motion of the complex) and $\tau_{v}$ (correlation time of the transient ZFS)(compare Eq. 7). Until now, most of the literature derives its information on electronic relaxation indirectly, through nuclear magnetic relaxation dispersion (NMRD) experiments ${ }^{36} 41$. The 


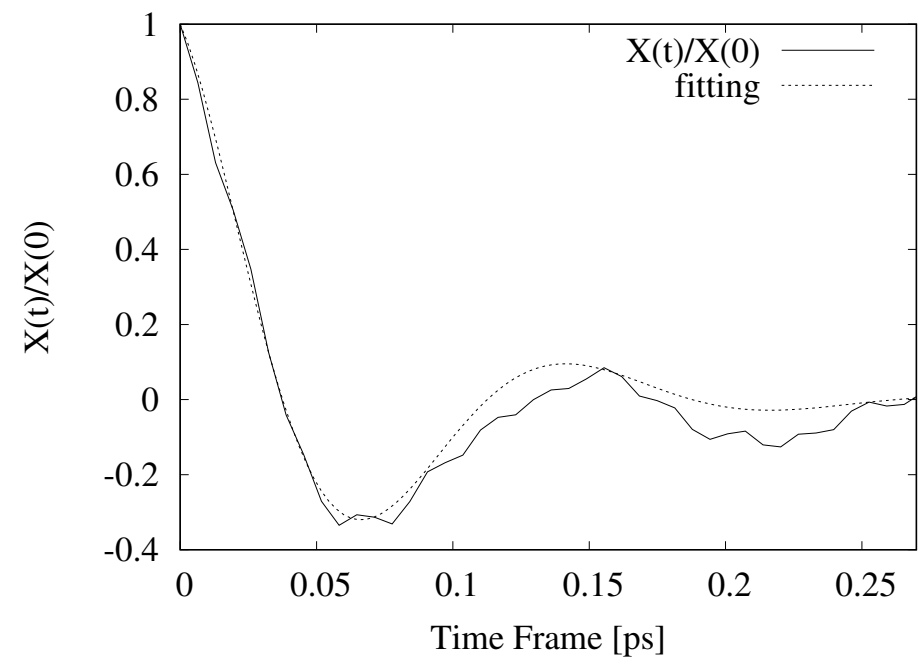

FIG. 11. Normalized TCF for $\mathrm{X}(\mathrm{t})=\langle(D(0)-\langle D\rangle) *(D(t)-\langle D\rangle)\rangle$ of the deviation in the $D$ parameter from the CASSCF calculations showing the combined average over the four blocks and the standard error.

derivation of these parameters and fitting of the data usually has several assumptions and so must be approached with a bit of caution. Therefore, it is essential to gather accurate qualitative information on the values associated with the ZFS from first-principles. In our study we assessed some of the paramagnetic parameters using both CASSCF and DFT.

In particular a better approximation of experimental values of the static ZFS was derived from the CASSCF method which is on the same scale as experiment whereas the TPSS functional in DFT is off by a factor of 10. From our CASSCF calculation, the correlation time of the transient ZFS obtained was on the sub-picosecond time scale, shorter than those derived from experimental data. However, it must be noted that there was a higher correlation between the individual elements of the $d_{i j}$ matrix in the case of CASSCF. The faster time scale also leads to the conclusion that electron spin relaxation in Gd(III) complexes is largely modulated by the much slower rotation of the static contribution. The dynamics of the transient ZFS indeed can be modeled by a Gaussian process as concluded by Lasoroski et $a \frac{19}{19}$. Moreover, it was found that across the AIMD snapshots there is little correlation between the elements of the transient ZFS between CASSCF and DFT. There is only a weak correlation between CASSCF and DFT when it comes to the $D$ parameter. It is important to notice that the time constants are similar between DFT and CASSCF, since the underlying MD is the same, but that this choice of DFT is not well correlated with CASSCF. 
Due to the lack of agreement of any of the DFT methods with the CASSCF results, it is not meaningful in the present study to evaluate the Couple-Perturbed $(\mathrm{CP})^{31}$ and PedersonKhanna(PK $)^{32}$ formalisms against each other. For a discussion about the deficiencies within each of the DFT methods we refer to the paper by Lasoroski et al. 19 .

By using the LC-BLYP functional we were able to obtain a static $D_{S}$ parameter that was much closer to the results from CASSCF and derived from experiment. However, the ZFS as computed using the LC-BLYP functional is not correlated with the CASSCF method. This shows that further combined studies of the ZFS of gadolinium complexes with both CASSCF and DFT are necessary to establish accurate and yet computationally economical methods for ZFS calculations. For extensive ZFS sampling, we require methods which are as efficient as DFT which can handle tens of thousands of configurations. On the other hand, methods such as CASSCF are more reliable and important for evaluating different approximations which allow for extensive sampling. Therefore, when both methods are used in tandem one can get an accurate estimation of the level of error that one is facing in each step of the process.

\section{ACKNOWLEDGMENTS}

We acknowledge financial support from the Marie-Curie ITN, and the Swedish Research Council. The research leading to these results has received funding from the People Pro-

gramme (Marie Curie Actions) of the European Union's Seventh Framework Programme FP7/2007-2013/ under REA grant agreement $n^{\circ} 317127$. The computations were performed on resources provided by the Swedish National Infrastructure for Computing (SNIC) at the Swedish National Supercomputer Center (NSC), the High Performance Computer Center North (HPC2N), and Chalmers Centre for Computational Science and Engineering (C3SE).

\section{APPENDIX}

\section{REFERENCES}

${ }^{1}$ S. Aime, M. Botta, and E. Terreno, Adv. Inorg. Chem. 57, 173 (2005).

${ }^{2}$ P. Caravan, Chem. Soc. Rev. 35, 512 (2006).

${ }^{3}$ C. F. G. C. Geraldes and S. Laurent, Contr. Med. Mol. Imag. 4, 1 (2009). 


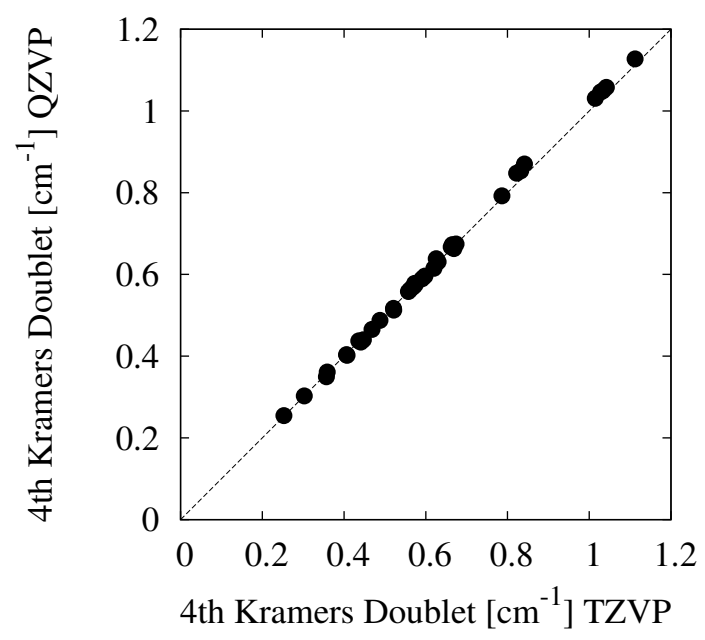

FIG. 12. The correlation in the energy level of the $4^{\text {th }}$ Kramer doublets using QZVP and def2TZVPP basis sets sampled over the AIMD trajectory.

${ }^{4}$ N. Bloembergen and L. O. Morgan, J. Chem. Phys. 34, 842 (1961).

${ }^{5}$ A. Merbach S., L. Helm, and E. Toth, eds., The chemistry of contrast agents in medical magnetic resonance imaging, 2nd ed. (Wiley, 2013).

${ }^{6}$ S. Rast, A. Borel, L. Helm, E. Belorizky, P. H. Fries, and A. E. Merbach, J. Am. Chem. Soc. 123, 2637 (2001).

${ }^{7}$ P. H. Fries and E. Belorizky, ChemPhysChem 13, 2074 (2012).

${ }^{8}$ E. Belorizky and P. H. Fries, Phys. Chem. Chem. Phys. 6, 2341 (2004).

${ }^{9}$ M. Rubinstein, A. Baram, and Z. Luz, Mol. Phys. 20, 67 (1971).

${ }^{10}$ P.-O. Westlund, N. Benetis, and H. Wennerström, Mol. Phys. 61, 177 (1987).

${ }^{11}$ M. Odelius, C. Ribbing, and J. Kowalewski, J. Chem. Phys. 103, 1800 (1995).

${ }^{12}$ M. Odelius, C. Ribbing, and J. Kowalewski, J. Chem. Phys. 104, 3181 (1996).

${ }^{13}$ D. Kruk and J. Kowalewski, J. Chem. Phys. 116, 4079 (2002).

${ }^{14}$ J. Mareš, H. Liimatainen, K. Laasonen, and J. Vaara, J. Chem. Theory Comput. 7, 2937 (2011).

${ }^{15}$ J. Mareš, H. Liimatainen, T. O. Pennanen, and J. Vaara, J. Chem. Theory Comput. 7, 3248 (2011).

${ }^{16}$ J. Rantaharju, J. Mareš, and J. Vaara, J. Chem. Phys. 141, 014109 (2014),

${ }^{17}$ J. Rantaharju and J. Vaara, Phys. Rev. A 94, 043413 (2016).

${ }^{18}$ J. Vaara, S. A. Rouf, and J. Mareš, J. Chem. Theory Comput. 11, 4840 (2015). 
${ }^{19}$ A. Lasoroski, R. Vuilleumier, and R. Pollet, J. Chem. Phys. 141, 014201 (2014).

${ }^{20}$ D. Delli Castelli, M. C. Caligara, M. Botta, E. Terreno, and S. Aime, Inorg. Chem. 52, 7130 (2013).

${ }^{21}$ S. Khan, A. Kubica-Misztal, D. Kruk, J. Kowalewski, and M. Odelius, J. Chem. Phys. 142, 034304 (2015).

${ }^{22}$ F. Neese, Wiley Interdisc. Rev. - Comput. Molec. Sci. 2, 73 (2012).

${ }^{23}$ E. v. Lenthe, E. J. Baerends, and J. G. Snijders, J. Chem. Phys. 99, 4597 (1993).

${ }^{24}$ B. A. Hess, C. M. Marian, U. Wahlgren, and O. Gropen, Chem. Phys. Lett. 251, 365 (1996).

${ }^{25}$ F. Weigend and R. Ahlrichs, Phys. Chem. Chem. Phys. 7, 3297 (2005).

${ }^{26}$ A. Schäfer, C. Huber, and R. Ahlrichs, J. Chem. Phys. 100, 5829 (1994).

${ }^{27}$ D. A. Pantazis and F. Neese, J. Chem. Theory Comput. 5, 2229 (2009).

${ }^{28}$ K. Eichkorn, F. Weigend, O. Treutler, and R. Ahlrichs, Theo. Chem. Acc. 97, 119 (1997).

${ }^{29}$ B. A. Hess, Phys. Rev. A 32, 756 (1985).

${ }^{30}$ D. Ganyushin and F. Neese, J. Chem. Phys. 125, 024103 (2006).

${ }^{31}$ F. Neese, J. Chem. Phys. 127, 164112 (2007).

${ }^{32}$ M. R. Pederson and S. N. Khanna, Phys. Rev. B 60, 9566 (1999).

${ }^{33}$ R. Maurice, C. De Graaf, and N. Guihéry, Phys. Chem. Chem. Phys. 15, 18784 (2013).

${ }^{34}$ A. Savin and H.-J. Flad, Int. J. Quant. Chem. 56, 327 (1995).

${ }^{35}$ P. M. W. Gill and R. D. Adamson, Chem. Phys. Lett. 261, 105 (1996).

${ }^{36}$ D. H. Powell, O. M. N. Dhubhghaill, D. Pubanz, L. Helm, Y. S. Lebedev, W. Schlaepfer, and A. E. Merbach, J. Am. Chem. Soc. 118, 9333 (1996).

${ }^{37}$ S. Aime, S. G. Crich, E. Gianolio, E. Terreno, A. Beltrami, and F. Uggeri, Europ. J. Inorg. Chem. , 1283 (1998).

${ }^{38}$ F. Uggeri, S. Aime, P. L. Anelli, M. Botta, M. Brocchetta, C. de Haën, G. Ermondi, M. Grandi, and P. Paoli, Inorg. Chem. 34, 633 (1995).

${ }^{39}$ S. Aime, M. Botta, G. Ermondi, E. Terreno, P. L. Anelli, F. Fedeli, and F. Uggeri, Inorg. Chem. 35, 2726 (1996).

${ }^{40}$ H. Lammers, F. Maton, D. Pubanz, M. W. Van Laren, H. Van Bekkum, A. E. Merbach, R. N. Muller, and J. A. Peters, Inorg. Chem. 36, 2527 (1997).

${ }^{41}$ C. F. G. C. Geraldes, A. D. Sherry, I. Lazar, A. Miseta, P. Bogner, E. Berenyi, B. Sumegi, G. E. Kiefer, K. McMillan, F. Maton, and R. N. Muller, Magn. Reson. Med. 30, 696 
(1993). 